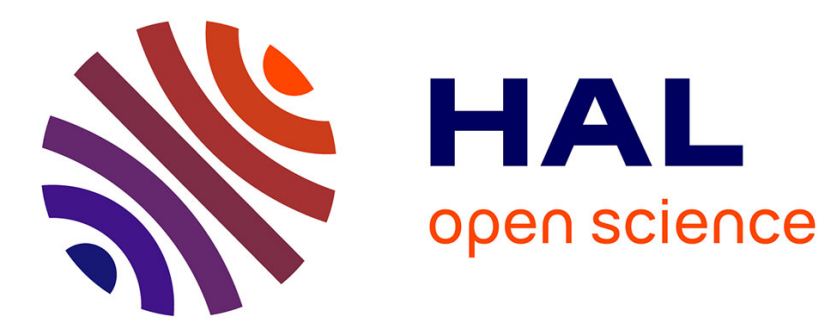

\title{
The clinical use of IDH1 and IDH2 mutations in gliomas
}

\author{
Alberto Picca, Giulia Berzero, Anna Luisa Di Stefano, Marc Sanson
}

\section{To cite this version:}

Alberto Picca, Giulia Berzero, Anna Luisa Di Stefano, Marc Sanson. The clinical use of IDH1 and IDH2 mutations in gliomas. Expert Review of Molecular Diagnostics, 2018,18 (12), pp.1041-1051. 10.1080/14737159.2018.1548935 . hal-01971809

\section{HAL Id: hal-01971809 \\ https://hal.sorbonne-universite.fr/hal-01971809}

Submitted on 7 Jan 2019

HAL is a multi-disciplinary open access archive for the deposit and dissemination of scientific research documents, whether they are published or not. The documents may come from teaching and research institutions in France or abroad, or from public or private research centers.
L'archive ouverte pluridisciplinaire HAL, est destinée au dépôt et à la diffusion de documents scientifiques de niveau recherche, publiés ou non, émanant des établissements d'enseignement et de recherche français ou étrangers, des laboratoires publics ou privés. 


\title{
The clinical use of IDH1 and IDH2 mutations in gliomas
}

\author{
Alberto Picca ${ }^{a}$, Giulia Berzero ${ }^{b, c}$, Anna Luisa Di Stefano ${ }^{d, e}$ and Marc Sanson ${ }^{d, f}$
}

a Neuroscience Consortium, University of Pavia, Italy; ${ }^{b}$ Neuroncology Unit, IRCCS Mondino Foundation, Pavia, Italy; 'Biomedical Sciences, University of Pavia, Italy; dSorbonne Universités, Paris, France; 'Department of Neurology, Foch Hospital, Suresnes, Paris, France; fService de Neurologie 2, AP-HP,

Hôpital de la Pitié-Salpêtrière, Paris, France

ABSTRACT

Introduction: Mutations in the genes isocitrate dehydrogenase (IDH) 1 and 2 have been reported in a limited number of tumors. In gliomas, IDH mutations are primarily detected in WHO grade II-III tumors and represent a major biomarker with diagnostic, prognostic, and predictive implications. The recent development of IDH inhibitors and vaccines suggests that the IDH mutation is also an appealing target for therapy.

Areas covered: This review focuses on the role of IDH mutations in diffuse gliomas. Besides discussing their role in gliomagenesis, we will emphasize the role of IDH mutations in clinical practice as a diagnostic, prognostic and predictive biomarker, and as a potential therapeutic target. Noninvasive detection of the IDH mutation by means of liquid biopsy and MR spectroscopy will also be discussed. Expert commentary: While IDH mutation is a consolidated diagnostic and prognostic biomarker in clinical practice, its role in oncogenesis is far from being elucidated, and there are several pending issues. The routine use of noninvasive techniques for detection and monitoring of the IDH status remains challenging. Although the IDH mutation is a very early alteration in gliomagenesis, it may then be omitted during tumor progression. This observation has important implications when designing targeted clinical trials.

\author{
KEYWORDS \\ IDH1and IDH2 mutations; \\ gliomagenesis; grade II and \\ III gliomas; biomarkers; \\ target therapy
}

\section{Introduction}

Isocitrate dehydrogenase (IDH) mutations are hotspot mutations affecting the genes that encode for the isocitrate dehydrogenase 1 (IDH1, cytoplasmic isoform) or 2 (IDH2, mitochondrial isoform) enzyme, all resulting in amino acid substitution at the active site and finally in a neomorphic activity that leads to profound modifications in the epigenetic profile of the cell.

IDH mutations have been first identified in diffuse gliomas in 2009 [1,2]. Since their discovery, IDH mutations have acquired an increasingly prominent role in diffuse gliomas management, as they bear diagnostic, prognostic, and predictive implications. They now represent the cornerstone of glioma classification and prognostic stratification, and they are actively investigated as a potential actionable target.

Mutations in the IDH genes have subsequently been identified in other tumor types, including acute myeloid leukemia $\left(I D H 1^{R 132}, I D H 2^{R 140}\right.$, and $\left.I D H 2^{R 172}\right)$ [3], cholangiocarcinomas $\left(I D H 1^{R 132}\right.$ and $\left.I D H 2^{R 172}\right)$ [4], chondromas and chondrosarcomas $\left(I D H 1^{R 132}\right.$ and $\left./ D H 2^{R 172}\right)$ [5], angioimmunoblastic T-cell lymphoma $\left(I D H 2^{R 172}\right)[6]$, melanomas $\left(I D H 1^{R 132}\right)$ [7], and a rare subtype of breast cancer (solid papillary carcinoma with reverse polarity, $I D H 2^{R 172}$ ) [8]. Clinical trials on IDH inhibitors are ongoing in these populations as well.

The present review will focus on the role of IDH mutations in diffuse gliomas. Besides analyzing the role of IDH mutations in gliomagenesis, we will discuss the use of IDH mutations in clinical practice as a diagnostic, prognostic, and predictive biomarker, as well as an actionable target. Emerging techniques for noninvasive detection of the IDH status will be described, with reference to their limitations and benefits in daily practice. Lastly, perspectives and future developments will be illustrated.

\subsection{IDH mutation, D2HG production, and gliomagenesis}

IDH mutations concern most of lower (i.e. II and III) grade gliomas and secondary glioblastomas, and only $5 \%$ of - apparently - primary glioblastomas [9].

Mutations in the IDH genes affect the codon 132 of IDH1 $\left(\mathrm{IDH} 1^{R 132}\right.$, in about $90 \%$ of cases) or the codon 172 of IDH2 $\left(I D H 2^{R 172}\right.$, in about $10 \%$ of cases) [10], in a mutually exclusive way. In both cases, the hotspot mutation results in amino acid substitution at a key residue of the encoded protein. IDH2 ${ }^{\mathrm{R} 140}$ mutations, common in acute myeloid leukemia [11], have not been reported in gliomas [12]. Mutations arising in $\mathrm{IDH} 1^{\mathrm{G} 97}$ $[13,14], \quad I D H 1^{R 100}$ [14-16] (the homologous of $\mathrm{IDH} 2^{\mathrm{R} 140}$ ), $\mathrm{IDH} 1^{\mathrm{R} 109}$ [17], and $\mathrm{IDH} 1^{\mathrm{R} 314}$ [18] have been anecdotally reported in gliomas.

IDH1 and IDH2 are enzymes that physiologically convert isocitrate to a-ketoglutarate ( $a-K G)$. These two enzymes have similar function but different cell compartmentalization: IDH1 is located in the cytosol, while IDH2 in mitochondria. Mutations in either IDH1 or IDH2 result in a neomorphic enzymatic activity, causing the stereospecific conversion of a-KG 
(produced by the wild type enzyme) to the D-enantiomer of 2-hydroxyglutarate (D2HG), that in turn accumulates within tumor cells $[19,20]$. IDH mutations are virtually always heterozygous mutations. The presence of a wildtype copy of the gene is necessary to provide the substrate for the mutant enzyme to produce the D2HG [21]. Loss of the wildtype allele, which may occur during tumor progression, also reduces the neomorphic activity [22].

D2HG acts as an oncometabolite and it is supposed to be the main driver of gliomagenesis. Because of its structural affinity to aKG, D2HG competitively inhibits several a-KG-dependent dioxygenases (as the JmjC domain-containing family of histone demethylases [23-25] and the TET family of methylcytosine hydroxylases $[25,26]$ ) with profound consequences on the epigenetic regulation of the cell. The enzymatic blockade induced by the accumulation of D2HG results in increased levels of histone $\mathrm{H} 3$ lysine methylation levels [24] and in global DNA hypermethylation (glioma CpG island methylator phenotype, G-CIMP) [26-28]. Of note, histone H3.3 mutations, frequently found in pediatric gliomas [29,30], and TET2 mutations, frequently found in myeloid malignancies [31], are mutually exclusive with IDH mutations $[26,29,30,32]$, suggesting that they have overlapping roles in tumorigenesis. The recently developed DNA methylation-based classification of central nervous system tumors [33] clearly shows that IDH mutant gliomas represent a well-defined methylation class, strongly separated from the nonmutant counterpart. In TGCA gene expression-based molecular classification of glioblastomas, IDH mutation strongly relates with the Proneural subtype [34].

Of interest, two different inborn error of metabolism can cause the pathological accumulation of one of the 2HG enantiomers, conditions called respectively D- and L-2HG aciduria. Strikingly, an increased risk of malignant brain tumors has been reported for L2HG aciduria [35-37], but not for D2HG aciduria [38].

While IDH mutations profoundly modify gene expression, a direct oncogenic effect has not been clearly demonstrated. Induced expression of $\mathrm{IDH} 1^{\mathrm{R} 132 \mathrm{H}}$ mutations in CNS cells of mouse models failed to induce glioma formation [39,40]. IDH mutation and the subsequent epigenetic remodeling result in a block of differentiation [24] that acts as a fertile substrate in which, over time, the occurrence of new 'lineage-defining' alterations (i.e. $1 p / 19 q$ codeletion, CIC, FUBP1, and pTERT mutations in oligodendrogliomas; p53 and ATRX mutations in astrocytomas) lead to gliomagenesis [41-43]. Succeeding 'tertiary' alterations (activating mutations of intracellular signaling pathways or amplification/activation of receptor tyrosine kinases) may then occur and lead to tumor progression to higher grades [44].

It is likely that the IDH mutation is not needed along the whole natural history of the tumor. At the latest stages of the disease, IDH mutation is no longer a driver alteration for a large number of gliomas, and becomes a passenger mutation while tertiary driver alterations appear. Johannesen et al. [45] . showed that the IDH mutant enzyme is not required for tumor maintenance after the occurrence of tertiary oncogenic alterations. Consistently, Mazor et al. [46] identified in recurrent gliomas IDH1 copy number alterations (CNA) resulting in impaired D2HG production. In vivo and in vitro models showed clonal selection for CNA, suggesting an advantage from losing IDH mutant function once tertiary alterations are established [46]. These findings suggest a potential benefit from loss of mutated function in advanced stages of IDH mutant gliomas. These observations still need further confirmation but could affect the development of target therapies directed to IDH mutant blockade.

Recent studies added further elements to this scenario, showing that the IDH mutation-driven methylator phenotype can directly produce oncogenic signals by reshaping the threedimensional (3D) chromatin structure. Flavahan et al. [47] showed that DNA hypermethylation in specific sites reduces its binding with CCCTC-binding factor (CTCF), an insulator protein, modifying therefore the 3D structure of the chromatin; this leads to disruption of genome topological domain boundaries. PDGFRA, an important oncogene, was aberrantly activated through interaction with a constitutive enhancer physiologically relegated in a different domain [47]. Similarly, in IDH mutant astrocytes, the repression of SOX2, a condition that acts as an early driver of gliomagenesis, is due to the hypermethylation of DNA binding sites for the chromatin insulator CTCF, leading to the dissociation of the SOX2 promoter from critical enhancer elements [48]. These two significant examples illustrate the pleiotropic and complex effects of IDH mutation on gene regulation.

The D2HG released in the microenvironment may also alter the functioning of peritumoral non-neoplastic cells, such as neurons and immune cells. The presence of IDH mutation has been linked with an increased risk of seizures in gliomas [49]. D2HG is structurally similar to glutamate and activate NMDA receptors in in vitro models [50,51], and may cause abnormal discharges in infiltrated neurons. More importantly, recent works have shown that IDH status also modulates the tumor-associated immune system. Compared to their wildtype counterpart, IDH mutant gliomas show reduced immune infiltrates [52-55] for both CD4 + and CD8+ lymphocytes [55]. It has been first speculated that this could in part explain the better outcome of IDH mutant gliomas, as immune infiltration is generally linked to poorer outcome [56] 8. In fact there are now direct evidences showing that D2HG accumulation exerts immunosuppressive effects. Activated CD4+ and CD8 + T cells proliferation is decreased in presence of high level D2HG [55]. D2HG reduces both the expression of several chemokines (such as CXCL10) and T cells migration in presence of chemoattractants, leading to a reduced immune infiltration [52,54,55]. Importantly, this immunosuppressive effect is reversed by IDH inhibitors. Moreover, the D2HG accumulation impairs complement-mediated tumor cell killing with both classical and alternative pathways [55]. IDH mutant tumors can also acquire mechanisms of resistance to natural killer cells by silencing the expression of the NKG2D-ligands ULBP1 and ULBP3 [57]. Interestingly, this could be antagonized by decitabine, an hypomethylating treatment which in vitro restored expression of NKG2D ligands. Very recently it has been shown that D2HG released by tumor cells is taken up by $\mathrm{T}$ cells and impairs their function. D2HG disrupt transcription and nuclear translocation of nuclear factor of activated T cells and promotes polyamine synthesis, finally resulting in reduced ATP/ADP levels and thus suppression of $T$ cell activity and proliferation [58]. All these mechanisms contribute to severely affect the antitumor immune response. On the other hand, the expression of the immune checkpoint protein PD1, which is an important mechanism of tumor escape [59], is 
repressed in IDH mutant gliomas by promoter methylation. This may participate to the resistance to immune checkpoint inhibitors treatments [60].

It is getting also more and more likely that not all the IDH mutation effects are mediated by the D2HG. The activity of IDH mutant enzyme leads to reduced levels of NADPH [61,62]. Seen its prominent role in protection from reactive oxygen species, this metabolic alteration may explain the enhanced radiosensitivity of IDH mutated gliomas.

\subsection{The diagnostic and prognostic role of IDH mutation in diffuse gliomas}

There is now solid evidence that IDH mutations have a major diagnostic and prognostic role in diffuse gliomas [9,63-67]. On these bases, the IDH status has been incorporated in the 2016 revision of the WHO classification of Tumors of the Central Nervous System as a defining feature for different tumor entities [68]. The codeletion of chromosomes $1 p$ and $19 q$, that invariably associates with the IDH mutation [63], is the other molecular alteration included into the classification of diffuse gliomas. The combination of the IDH and $1 p / 19 q$ codeletion status allows to distinguish three distinct molecular subgroups: (1) IDH mutant, 1p/19q codeleted gliomas (corresponding to grade II and III oligodendrogliomas); (2) IDH mutant, $1 p / 19 q$ non-codeleted gliomas (corresponding to grade II and III astrocytomas and secondary glioblastomas); (3) IDH wildtype gliomas (primary glioblastomas and a minority of diffuse grade II and III astrocytomas). The prognostic stratification based on these molecular subgroups accounts better than the classification based on the histological appearance alone for the biological behavior of the neoplasm [65,69]. IDH mutant, 1p/19q codeleted gliomas harbor the better prognosis, with a median survival of 15 years for both grade II and III. IDH mutant, non-codeleted gliomas have a median survival of 7-9 years for grade II and 5-6 years for grade III. IDH wildtype lower grade gliomas have the poorest prognosis, approaching glioblastoma median survival (2 years for grade III, 3.5 years for grade II) [63,66,67].

While grading (II vs. III) has a modest impact in IDH mutant gliomas [70], the loss of CDKN2A/B on 9p21, the presence of necrosis and for astrocytomas the total number of copy number variation are independent prognostic factor and may be used to define an algorithm that is superior to histological grading in predicting the overall survival [71-73].

The IDH mutation also predicts the response to cytotoxic treatments. IDH mutant gliomas are more sensible to radiotherapy, as they have reduced levels of NADH and NADPH $[61,62]$. The pharmacological inhibition of IDH restores NADH and NADPH levels and reduces the cytotoxic effect of ionizing radiations on tumor cells [62]. These data suggest that the administration of IDH inhibitors during radiotherapy might decrease the cytotoxic effects of radiation.

IDH mutant tumors also display improved response to chemotherapy: randomized clinical trials conducted in patients with high risk grade II [74] and grade III $[75,76]$ gliomas treated with radiotherapy showed that only patients with IDH mutant tumors clearly benefit from the addition of adjuvant PCV (procarbazine-CCNU-vincristine) chemotherapy to radiation. IDH mutant gliomas have a reduced expression of O6-methylguanine DNA methyltransferase gene, a known DNA repair enzyme associated to chemosensitivity [77], via promoter hypermethylation [28]. Moreover, D2HG competitively inhibits several aKG-dependent dioxygenases involved in DNA repair, as the AlkB family $[78,79]$ and KDM4A/B enzymes [80]. All these observations may explain the higher sensitivity of IDH mutant tumors to alkylating agents.

\subsection{IDH status assessment: immunohistochemistry and sequencing}

The IDH genotype is assessed by sequencing of tumor DNA extracted from fresh frozen or formalin-fixed paraffinembedded tissue. The most common sequencing technique to assess the IDH status remains the Sanger method, as it is a widely used and low-cost technique. However, Sanger sequencing can miss IDH mutations when mutant allele levels are below 15-20\%; this primarily occurs when the tumor sample is heavily contaminated by normal cells, as it occurs in peripheral surgical sampling.

Pyrosequencing is a quantitative DNA sequencing technique based on the detection of pyrophosphate released during nucleotide incorporation ('sequencing by synthesis') that can identify IDH1/2 mutant allele frequency as low as $5 \%[81,82]$. Real-time PCR with post-PCR fluorescence melting curve analysis also showed higher analytical sensitivity compared to Sanger sequencing [83].

Newer sequencing techniques with higher sensitivity have been developed during the last few years. PCR clamping/ Amplification Refractory Mutation System [84] and COamplification at Lower Denaturation temperature PCR (COLD$P C R$ ) [85] are based on the preferential amplification of the mutant allele and may detect mutant IDH allele levels below 5\%. Droplet digital PCR [86] relies on DNA partitioning in nanoliter-sized droplets, which then carries out a $P C R$ reaction with a single-molecule resolution.

The IDH status can alternatively be assessed using multiplexed, high-throughput genotyping platforms [87,88], and deep next generation sequencing panels [89-92]. These panels allow the contemporary acquisition of a wide panel of genetic alterations, including mutations, amplifications, and deletions.

As IDH mutant tumors express a DNA hypermethylator profile $[27,28]$, IDH status can be inferred from tumor DNA profiling on 450 and $850 \mathrm{k}$ arrays.

Immunohistochemistry is routinely performed as part of the histopathological assessment. As mutations in the IDH1 gene consist in more than $90 \%$ of cases in an arginine-tohistidine substitution at residue $132[10]^{9}$, the use of a specific antibody that binds the mutant $\mathrm{IDH} 1^{\mathrm{R} 132 \mathrm{H}}$ protein allows to identify mutant tumor cells by immunohistochemistry in most cases $[93,94]$. This method can identify single mutated cells over a background of normal cells, with a sensitivity that may overcome standard sequencing [95]. However, non-R132H IDH1 mutations and IDH2 mutations are missed. In case of immunohistochemistry negativity and with the suspicion of an IDH mutation based on patient age (patients with glioblastomas younger than 55 years) and/or 
histological features (e.g. astrocytoma phenotype, loss of ATRX expression, oligodendroglial phenotype, or $1 p 19 q$ codeletion), IDH1 and IDH2 sequencing should be performed. Sequencing can be omitted in case of elderly patients (i.e. $>55$ years old) with an histological appearance of glioblastoma, or an immunostaining positivity for the H3K27M mutation, as the two genetic alterations are known to be mutually exclusive [29].

\subsection{Noninvasive detection and monitoring of the IDH mutations}

Further from well-defined IDH testing from tumor specimen, there is a strong need for less-invasive testing, for several reasons: in primis, not all gliomas are amenable to resection, and the identification of IDH mutation could permit the diagnosis without the surgical risk of a biopsy; furthermore, all biopsies are burdened by the possibility of sampling error, and could underscore the IDH status or even the glioma diagnosis; the identification of a quantifiable biomarker could eventually help in evaluating the response to therapies, in differential diagnosis with pseudoprogressions and pseudorelapses, and in predicting relapses before the neuroimaging. Lastly, it has been suggested that the IDH mutation drive the prognostic benefit associated with a greater extent of resection [96], so knowing the IDH status would be of interest also in the preoperative planning. On this regard, PCR $[97,98]-$, desorption electrospray mass spectroscopy $[99,100]$, matrix-assisted laser desorption ionization-time of flight mass spectroscopy [101], and optical spectroscopy [102,103]-based techniques have been developed for rapid detection of IDH mutation or D2HG accumulation in the intraoperative setting.

A promising biomarker for IDH mutant tumors is D2HG, as it is produced at detectable concentrations in tumor tissues only [19], and its level has been correlated with response to IDH inhibitors [104] in preclinical models.

Tumor D2HG millimolar levels can be detected in vivo with magnetic resonance spectroscopy [105-107]. Identification of the metabolite is diagnostic for IDH mutation, as it is absent in normal brain tissue. However, standard PRESS sequences are burdened by up to $26 \%$ of false positive due to overlapping with the signal from other metabolites of normal brain (as glutamate and glutamine) at spectrum location of 2.25 ppm [107]. Several techniques have been experimented to overcome the limit and optimize D2HG detection, as TE 97 ms optimized PRESS [106], 2D correlation spectroscopy [105], and optimized semiLASER [108] (see Leather et al. [109] for review). Edited MRS (based on detection of D2HG signal at $4.02 \mathrm{ppm}$, using MEGA-PRESS sequence) recently showed being more reliable than optimized PRESS MRS in an extended series of patients, reaching an optimal $100 \%$ sensitivity, specificity, PPV, and NPV for diagnosis [110].

Two series evaluated D2HG-detecting MRS (with optimized PRESS sequences) in a clinical setting, with different results in terms of sensitivity (48-89.5\%) and specificity (81.3-100\%) $[111,112]$. Sensitivity appeared to be mostly influenced by tumor dimension [111], while low specificity were attributed to inclusion of necrotic areas [112], which presence is however uncommon in IDH mutant gliomas and should raise the suspicion of a false positive result. One may speculate that this may be due to the production of L2HG by hypoxia [113], as spectroscopy technique is unable to differentiate the two enantiomers.

Other than diagnostic, D2HG-detecting MRS can also be helpful in longitudinal follow up, as D2HG signal decrease in correlation with treatment response $[111,114,115]$.

As D2HG-detecting MRS is not widely diffused, other papers explored the utility of standard and advanced MRI techniques in predicting IDH status. IDH mutant gliomas usually are better defined, poorly or not enhancing, lobar tumors, while IDH wildtype gliomas present a greater percentage of non-lobar and multifocal locations [116]. In diffusion and perfusion sequences, IDH mutation is associated with higher apparent diffusion coefficient and lower regional cerebral blood volume values [117-119]. The 'T2FLAIR mismatch' sign (i.e. a completely hyperintense signal in T2 sequences corresponding to an hypointensity with hyperintense rim in fluid attenuated inversion recovery [FLAIR] sequences) is highly specific, though less sensitive, for IDH mutant, 1p/19q codeleted tumors [120,121, 122].

Another promising tool for poorly invasive prediction of IDH status is the detection of D2HG levels in body fluids. Differing from patients with AML [123] and cholangiocarcinoma [124] harboring IDH mutations, studies failed to find a detectable elevation of $\mathrm{D} 2 \mathrm{HG}$ in serum of patients with IDH mutant gliomas [125-127]; this may be attributed to the site-specificity of D2HG production. A further paper examined the D2HG levels in CSF and found a good specificity in detecting IDH mutation; however, the data were stronger for necrotic, contrast-enhancing higher grade gliomas compared to LGGs which represent the majority of IDH mutant gliomas [128]. Urinary D2HG levels in IDH mutant and wildtype gliomas have been also analyzed, but with discordant results [126,127].

A progressing field in oncology is the so called 'liquid biopsy,' which aims to detect tumor cells or tumor nucleic acids in body fluids $[129,130]$. Highly sensitive techniques as digital droplet PCR [131] or COLD PCR [85] can be useful to detect even very low levels of mutation. Boisselier et al. firstly reported the possibility to detect $\mathrm{IDH} 1^{\mathrm{R} 132 \mathrm{H}}$ mutation amplifying small-size tumor DNA from plasma of glioma patients [132]. In a recent work, Martinez-Ricarte et al. were able to define the mutational status of different genes, including IDH1/2, analyzing the CSF cell-free circulating tumor DNA. However, the technique lacked sensitivity in detecting ctDNA in three out of the five IDH mutant low grade gliomas in this study [133]. Another source of circulating tumor nucleic acids are extracellular vesicles (EV). Mutant IDH1 ${ }^{\mathrm{R} 132 \mathrm{H}}$ mRNA has been identified in CSF-, but not serum-, -derived EV of patients with mutated glioma [134].

Blood-brain barrier disruption (as reflected by contrast enhancement on MRI) and/or necrosis are associated with a higher amount of free circulating tumor DNA and higher probability to detect the mutation [132-134]. This appear as a limit as the majority of IDH mutant gliomas are nonenhancing gliomas, making the detection of tumor nucleic acids poorly sensitive in these entities. 


\subsection{IDH mutation as a theranostic marker}

\subsubsection{IDH inhibition}

As IDH mutation is an early, truncal and stable alteration leading to the production of a specific oncometabolite, the inhibition of the neomorphic enzymatic activity soon appeared as a logic therapeutic approach. Since the first report of an in vitro effective $\mathrm{IDH} 1^{\mathrm{R} 132 \mathrm{H}}$ inhibitor [104], several other inhibitors targeting also non-R132H IDH1 and IDH2 mutations have been developed [135-138].

Preclinical studies showed that IDH mutant inhibition cause a detectable decrease in D2HG levels and the induction of differentiation in IDH mutant glioma cells $[104,135,136]$. Kopinja et al. [136] . reported a decrease in 5-methylcytosine 435 levels in treated tumor sections compared to controls. However, no detectable effects on global DNA methylation levels were seen when directly analyzed [104,135]. A reduction of repressive histone trimethylation markers was identified in cells treated with higher doses of the inhibitor [104]. Murine models showed promising results in terms of tumor growth inhibition and increased survival [104,135,136,139]. However, IDH mutant inhibition did not result in mutant cells growth blockade in other studies, despite effective D2HG levels reduction, suggesting that in some lines tumor growth is independent from IDH mutant function $[136,140]$. As exposed above, IDH mutation at the late stages of glioma evolution is no longer driver nor necessary for tumor maintenance. Furthermore, number of epigenetic changes induced by D2HG are irreversible and remain fixed, even on withdraw of IDH mutation and D2HG [141]. Several phase I studies in humans have been developed in the last years. Final results of phase I trial of ivosidenib (AG-120) in AML have been recently published showing a favorable safety profile and encouraging response rate [142]. First data from phase I trials of AG-120 and AG-881 in gliomas has been presented in abstract form, showing a good safety profile and reporting prolonged disease control in a number of patients, mostly with non-contrast-enhancing tumors $[143,144]$. In line with the experimental data exposed above, this result may suggest that the inhibition of the neomorphic should be proposed at the early stage of the disease.

On the other hand, we may expect that the inhibition of neomorphic enzyme will trigger immune response. Indeed immunity to syngeneic IDH1 mutant tumors induced by IDH1specific peptide vaccination or checkpoint inhibition was improved by pharmacological blockade of the neomorphic enzymatic function of mutant IDH1 [58].

\subsubsection{Demethylating agents}

Since IDH mutation exerts most of its effect through DNA hypermethylation, the utility of demethylating drugs in gliomas has been explored. Decitabine and 5-azacytidine are two FDA-approved DNA methyltransferase (DNMT) inhibitors; in preclinical studies they both showed to reduce DNA hypermethylation and induce differentiation of glioma cells $[145,146]$. A phase I study with 5-azacytidine in hematological and solid tumors (including gliomas) is currently ongoing (clinicaltrials.gov identifier: NCT02223052).

\subsubsection{IDH vaccination}

The IDH mutation creates a tumor-specific, uniformly expressed neoantigen, theoretically suitable for targeted immunization. Murine models confirmed that vaccination with a peptide containing the mutated $\mathrm{IDH} 1^{\mathrm{R} 132 \mathrm{H}}$ epitope can elicit a mutationspecific acquired immune response $[147,148]$. The feasibility and effectiveness of IDH vaccines are currently explored in the human setting. The German NOA-16 [149] phase I trial of $\mathrm{IDH} 1^{\mathrm{R} 132 \mathrm{H}}$ vaccination in IDH mutant grade III-IV gliomas (clinicaltrials.gov identifier: NCT02454634) completed the data accrual and first results have been presented in abstract form. The induction of acquired humoral and/or T cell responses has been detected in most of the patients vaccinated [150]. Final results are expected for the end of the current year. The RESIST phase I trial of PEPIDH1M vaccine [151] in recurrent IDH mutant grade II gliomas (clinicaltrials.gov identifier: NCT02193347) is currently recruiting. In order to potentiate the effect of vaccination, the AMPLIFY-NEOVAC (EudraCT number:2017-000587-15) evaluate the combination of IDH1 mutation-specific peptide vaccination with PD-L1 checkpoint inhibition.

\subsubsection{Synthetic lethality}

Sulkowski et al. [80]. recently reported that IDH mutant cells harbor an intrinsic double-strand DNA break repair defect as a result of a deficit in homologous recombination (HR). D2HG induces $H R$ impairment by competitive inhibition of aKGdependent dioxygenases KDM4A/B. Pharmacological inhibition of the poly(ADP-ribose) polymerase (PARP), an enzyme implicated in HR pathways, induced a state of marked synthetic lethality in IDH mutant cells. IDH mutant cells sensitivity to PARP inhibition has been consistently shown in a different work [152]. Olaparib (an FDA-approved PARP inhibitor) administration in xenograft models caused a significant tumor growth delay [80]. Furthermore, olaparib enhanced temozolomide sensitivity of IDH mutant cells. Based on these results, a phase II study of olaparib in recurrent IDH mutant gliomas (OLAGLI) has been planned (clinicaltrials.gov identifier: NCT03561870).

There is recent evidence that IDH mutant cells have reduced expression of NAPRT1, a rate-limiting enzyme in NAD+-salvage pathways, via promoter hypermethylation. Tateishi et al. [140] demonstrated that blocking a different NAD+-salvage pathway via the inhibition of the NAMPT enzyme induces a strong reduction of intracellular NAD+ levels, impairment of tricarboxylic acid cycle and activation of metabolic-sensing pathway of autophagy, finally resulting in cell death. Testing on murine xenograft models confirmed a strong, IDH-dependent inhibition of tumor growth. NAMPT inhibitors have been reported as temozolomide sensitizers in glioblastoma cells [153], but to our knowledge no study has been performed to evaluate their utility in patients with IDH mutant gliomas.

Furthermore, IDH mutated tumors appear to be exquisitely sensitive to inhibitors of $\mathrm{BCl}-\mathrm{xL}$, an anti-apoptotic gene of the $\mathrm{Bcl}-2$ family. Tumors usually upregulate $\mathrm{Mcl}-1$ gene expression as an escape pathway from $\mathrm{BCl}-\mathrm{xL}$ inhibition; in IDH mutated tumors, D2HG downregulates $\mathrm{Mcl}-1$, inducing a state of 
synthetic lethality. Murine xenograft models confirmed a survival benefit from ABT263, a specific Bcl-xL inhibitor [154].

Lastly, D2HG production in IDH mutant gliomas is dependent on glutaminase enzymatic activity. Glutaminase is an enzyme involved in a-KG production pathway, hydrolyzing glutamine to glutamate. In vitro inhibition of glutaminase with a specific small molecule inhibitor selectively impaired tumor growth of IDH mutant, but not wildtype, tumor models. Intriguingly, D2HG levels remained stable, suggesting a different target of activity [155]. Of note, glioma cells may bypass glutaminase blocking via direct uptake of glutamate from the brain microenvironment $[156,157]$. A phase $1 \mathrm{~b}$ trial of a glutaminase inhibitor (CB-839) in association with temozolomide and radiotherapy has been planned (clinicaltrials.gov identifier: NCT03528642).

\section{Conclusion}

550 IDH status is the major classifier of diffuse gliomas and is now included in the WHO 2016 glioma classification. It is a major diagnostic, prognostic, predictive marker in gliomas-and possibly theranostic in the next future. It also represents an opportunity for non-invasive diagnosis based on liquid biopsy (D2HG measurement and/or IDH mutation detection) or MR spectroscopy. Because IDH mutation is the earliest and the most stable genetic alteration, and because it inhibits host immune response, IDH mutant tumors represents an ideal target for specific inhibitors and vaccination.

\section{Expert commentary}

The knowledge of the IDH status is mandatory for the management of glioma patients, as it impacts both patients prognosis and treatment choices. Widely available techniques easily performed in routine are nowadays available for IDH status determination on tissue samples.

An integrated diagnosis combining the $\mathrm{IDH} 1^{\mathrm{R} 132 \mathrm{H}}$ immunostaining and standard Sanger sequencing, when needed, is informative and sufficient in the vast majority of the cases. All results should always be interpreted taking into account sample provenience (tumor core versus invasion margins) and histological appearance in order to suspect possibly false negative results.

Several techniques with faster processing and higher analytical sensitivity compared to standard Sanger sequencing have been defined in the last years, but no one emerged as the new standard. They are usually chosen based on single laboratory expertise.

IDH mutation represents an opportunity for non-invasive diagnosis based on liquid biopsy (D2HG measurement and IDH mutation detection in body fluids) or MR spectroscopy. In contrast to other IDH mutant neoplasms, the liquid biopsy in IDH mutant gliomas still lacks sensitivity, probably due to the relatively small tumor mass, and also to the presence of an intact blood-brain barrier in low grade, non-enhancing gliomas which limits the free DNA -but not the D2HG- release. Overall mutant DNA or D2HG dosage appear more reliable and easier to detect in the CSF compared to the serum/ plasma. In parallel, several centers throughout the world have developed a reliable MR spectroscopy method to detect D2HG. However, none of these techniques is currently use in routine nowadays.

It still remains unclear why results with mutant IDH inhibitors have been less promising in gliomas than for myeloid leukemia. This is probably not due to the pharmacokinetics because we have several cases of patients non responder to IDH mutant inhibition in which MR spectroscopy clearly showed a decrease in D2HG levels (unpublished data). Alternatively, the reversibility of IDH mutant-induced epigenetic changes may also be dependent on tissue type, glial cells being possibly less prone to reverse epigenetic changes after D2HG withdrawal [141]. Importantly, as explained above, the acquisition of tertiary driver alterations over time, while IDH mutation becomes a passenger alteration (and may even be eliminated), suggest that maximal benefit for mutant IDHtargeted therapy occur when it is initiated early after initial diagnosis.

Finally, IDH mutations offer a unique opportunity for personalized treatments: these should be based on the deep knowledge of the pleiotropic effects of IDH mutation in tumor cell and microenvironment, and its role along the whole natural history of gliomas. There is therefore a need to better understand the consequences of IDH mutation: in first, the effects mediated by D2HG (on epigenetic and gene expression, particularly modifying the $3 \mathrm{D}$ folding of the chromatin but not only, because D2HG competitively inhibits a great variety of cellular enzymes), but also by different mechanisms (metabolic imbalances as the decrease of NADPH/NADP ratio). It also includes the paracrine effects of D2HG on the microenvironment (lymphocytes, macrophages, neurons). As a consequence, IDH inhibitors should be proposed early (see above). Secondly, IDH status uncover metabolic vulnerabilities that can be taken advantage to induce a specific synthetic lethal state, such as with PARP inhibition, NAD+ depletion, glutaminase inhibition. Finally IDH mutant gliomas may be excellent candidates for immunotherapeutic approaches: IDH mutant inhibitors showed to be able to disrupt the immunosuppressive paracrine effects of D2HG and can be combined with anti IDH mutant vaccination and/or checkpoint inhibitor therapies. Combined approaches appear thus promising and are likely to be one of the explored paths in the next years.

\section{Five-year view}

The last years have seen the affirmation of IDH mutation as the main prognostic and predictive marker in diffuse gliomas. The field is moving fast, and the next years will bring a better knowledge on the pleiotropic mechanism of the neomorphic IDH enzyme, exploring the effect of IDH mutation on epigenetic, gene expression and cellular metabolism, and the effect of D2HG on microenvironment and immune response. In addition, the recent identification of loci associated with the risk of developing specifically IDH mutant gliomas will also help to dissect the mechanism underlying IDH mutant gliomas, as compared to IDH wildtype $[158,159]$.

There is a need to improve sensitivity and specificity of non-invasive diagnosis of IDH mutant gliomas. The most 
reliable technique at the moment is the D2HG-detecting MR spectroscopy, which is likely to enter into clinical practice for preoperative evaluation in most centers; its utility in longitudinal follow up will be further defined.

The most important progresses are expected from new therapeutic approaches directed to the inhibition of or vaccination against the neomorphic enzyme, or taking advantage of synthetic lethality.

Based on positive safety results obtained from phase

655 I studies, several IDH inhibitors are going to be explored in phase II trials. Further from their use in monotherapy, they have promising synergistic effect with immunotherapy.

A deeper comprehension of mutated cells metabolism and their intrinsic vulnerabilities induced by D2HG accumulation 660 will lead to the development of new synthetic lethal drugs, while clinical trials will explore the feasibility and effectiveness of established ones.

\section{Key issues}

- Mutation of IDH1/2 genes is the first known genetic alteration in the vast majority of grade II and III diffuse gliomas, and lead to gliomagenesis mostly via the production of the oncometabolite D2HG and subsequent epigenetic remodeling.

- IDH mutation is the main molecular prognostic and predictive marker in diffuse gliomas.

- IDH mutation is a major classifier and is integrated in the 2016 revision of WHO classification of central nervous system tumors. Diffuse gliomas are classified into three main groups: IDH mutant - which in turn are separated into chromosomes $1 p / 19 q$ codeleted and not-codeleted - and IDH wildtype gliomas.

- IDH status determination is easy to assess in routine and includes the immunostaining for the most common $I D H 1^{R 132 H}$ mutationanddirect sequencing of codons IDH1

680 R132 and IDH2 R172 to detect the less frequent mutations.

- IDH mutation represents a unique opportunity for noninvasive diagnosis, based on mutation detection from free circulating DNA and/or D2HG detection by dosage (in the CSF) or by in vivo MR spectroscopy.

- IDH is a theranostic marker: innovative therapies are currently developed in clinical trials, directed toward specific inhibition of IDH mutant enzymes, specific vaccination against the mutant enzyme, or uncovering synthetic lethality, for example wit PARP inhibitors.

\section{Funding}

This paper was not funded.

\section{Declaration of interest}

The authors have no relevant affiliations or financial involvement with any organization orntity with a financial interest in or financial conflict with the subject matter or materials discussed in the manuscript. This includes employment, consultancies, honoraria, stock ownership or options, expert testimony, grants or patents received or pending, or royalties.

\section{Reviewers disclosure}

Peer reviewers on this manuscript have no relevant financial relationships or otherwise to disclose.

\section{References}

Papers of special note have been highlighted as either of interest $(\cdot)$ or of considerable interest (*) to readers.

1. Parsons DW, Jones $S$, Zhang $X$, et al. An integrated genomic analysis of human glioblastoma multiforme. Science. 2008;321:1807-1812.

2. Yan $H$, Parsons $D W$, Jin $G$, et al. $I D H 1$ and $I D H 2$ mutations in gliomas. N Engl J Med. 2009;360:765-773.

3. Mardis ER, Ding L, Dooling DJ, et al. Recurring mutations found by sequencing an acute myeloid leukemia genome. $\mathrm{N}$ Engl J Med. 2009;361:1058-1066.

4. Borger DR, Tanabe KK, Fan KC, et al. Frequent mutation of isocitrate dehydrogenase (IDH) 1 and IDH2 in cholangiocarcinoma identified through broad-based tumor genotyping. Oncologist. 2012;17:72-79.

5. Amary MF, Bacsi K, Maggiani F, et al. IDH1 and IDH2 mutations are frequent events in central chondrosarcoma and central and periosteal chondromas but not in other mesenchymal tumours. J Pathol. 2011;224:334-343.

6. Lemonnier F, Cairns RA, Inoue S, et al. The IDH2 R172K mutation associated with angioimmunoblastic T-cell lymphoma produces $2 \mathrm{HG}$ in $\mathrm{T}$ cells and impacts lymphoid development. Proc Natl Acad Sci USA. 2016;113:15084-15089.

7. Linos K, Tafe LJ. Isocitrate dehydrogenase 1 mutations in melanoma frequently co-occur with NRAS mutations. Histopathology. 2018 [cited 2018 Oct 22]; [Epub ahead of print]. DOI:10.1111/ his. 13707

8. Chiang S, Weigelt $\mathrm{B}$, Wen $\mathrm{H}-\mathrm{C}$, et al. IDH2 mutations define a unique subtype of breast cancer with altered nuclear polarity. Cancer Res. 2016;76:7118-7129.

9. Sanson M, Marie $Y$, Paris $S$, et al. Isocitrate dehydrogenase 1 codon 132 mutation is an important prognostic biomarker in gliomas. J Clin Oncol. 2009;27:4150-4154.

10. Horbinski C. What do we know about IDH1/2 mutations so far, and how do we use it? Acta Neuropathol. 2013;125:621-636.

11. Green $C L$, Evans $C M$, Zhao L, et al. The prognostic significance of IDH2 mutations in AML depends on the location of the mutation. Blood. 2011;118:409-412.

12. https://cancer.sanger.ac.uk/cosmic/gene/analysis?ln=IDH1.

13. Paugh BS, Qu C, Jones $C$, et al. Integrated molecular genetic profiling of pediatric high-grade gliomas reveals key differences with the adult disease. J Clin Oncol. 2010;28:3061-3068.

14. Ward PS, Cross JR, Lu C, et al. Identification of additional IDH mutations associated with oncometabolite R(-)-2-hydroxyglutarate production. Oncogene. 2012;31:2491-2498.

15. Pusch S, Sahm F, Meyer J, et al. Glioma IDH1 mutation patterns off the beaten track. Neuropathol Appl Neurobiol. 2011;37:428-430.

16. Gupta R, Flanagan S, Li CCY, et al. Expanding the spectrum of IDH1 mutations in gliomas. Mod Pathol. 2013;26:619-625.

17. Cruz GR, Dias Oliveira I, Moraes L, et al. Analysis of KIAA1549-BRAF fusion gene expression and IDH1/IDH2 mutations in low grade pediatric astrocytomas. J Neurooncol. 2014;117:235-242.

18. van Lith SAM, Navis AC, Lenting $K$, et al. Identification of a novel inactivating mutation in Isocitrate Dehydrogenase 1 (IDH1-R314C) in a high grade astrocytoma. Sci Rep. 2016;6:30486.

19. Dang L, White DW, Gross S, et al. Cancer-associated IDH1 mutations produce 2-hydroxyglutarate. Nature. 2009;462:739-744.

20. Ward PS, Patel J, Wise DR, et al. The common feature of leukemia-associated IDH1 and IDH2 mutations is a neomorphic enzyme activity converting alpha-ketoglutarate to 2-hydroxyglutarate. Cancer Cell. 2010;17:225-234.

21. Molenaar RJ, Radivoyevitch T, Maciejewski JP, et al. The driver and passenger effects of isocitrate dehydrogenase 1 and 2 mutations in 
oncogenesis and survival prolongation. Biochim Biophys Acta. 2014;1846:326-341.

22. Jin G, Reitman ZJ, Duncan CG, et al. Disruption of wild-type IDH1 suppresses D-2-hydroxyglutarate production in IDH1-mutated gliomas. Cancer Res. 2013;73:496-501.

23. Chowdhury R, Yeoh KK, Tian Y-M, et al. The oncometabolite 2-hydroxyglutarate inhibits histone lysine demethylases. EMBO Rep. 2011;12:463-469.

24. Lu C, Ward PS, Kapoor GS, et al. IDH mutation impairs histone demethylation and results in a block to cell differentiation. Nature. 2012;483:474-478.

25. Xu W, Yang H, Liu Y, et al. Oncometabolite 2-hydroxyglutarate is a competitive inhibitor of a-ketoglutarate-dependent dioxygenases. Cancer Cell. 2011;19:17-30.

26. Figueroa ME, Abdel-Wahab $\mathrm{O}$, Lu C, et al. Leukemic IDH1 and IDH2 mutations result in a hypermethylation phenotype, disrupt TET2 function, and impair hematopoietic differentiation. Cancer Cell. 2010;18:553-567.

27. Noushmehr $\mathrm{H}$, Weisenberger DJ, Diefes $\mathrm{K}$, et al. Identification of a CpG island methylator phenotype that defines a distinct subgroup of glioma. Cancer Cell. 2010;17:510-522.

28. Turcan S, Rohle D, Goenka A, et al. IDH1 mutation is sufficient to establish the glioma hypermethylator phenotype. Nature. 2012;483:479-483.

29. Schwartzentruber J, Korshunov A, Liu X-Y, et al. Driver mutations in histone $\mathrm{H} 3.3$ and chromatin remodelling genes in paediatric glioblastoma. Nature. 2012;482:226-231.

30. Sturm D, Witt $H$, Hovestadt V, et al. Hotspot mutations in H3F3A and IDH1 define distinct epigenetic and biological subgroups of glioblastoma. Cancer Cell. 2012;22:425-437.

31. Abdel-Wahab O, Mullally A, Hedvat C, et al. Genetic characterization of TET1, TET2, and TET3 alterations in myeloid malignancies. Blood. 2009;114:144-147.

32. Wu G, Broniscer A, McEachron TA, et al. Somatic histone H3 alterations in pediatric diffuse intrinsic pontine gliomas and non-brainstem glioblastomas. Nat Genet. 2012;44:251-253.

800 33. Capper D, Jones DTW, Sill M, et al. DNA methylation-based classification of central nervous system tumours. Nature. 2018;555:469-474.

34. Verhaak RGW, Hoadley KA, Purdom E, et al. Integrated genomic analysis identifies clinically relevant subtypes of glioblastoma characterized by abnormalities in PDGFRA, IDH1, EGFR, and NF1. Cancer Cell. 2010;17:98-110.

35. Moroni I, Bugiani M, D'Incerti L, et al. L-2-hydroxyglutaric aciduria and brain malignant tumors: a predisposing condition? Neurology. 2004;62:1882-1884.

36. Patay Z, Mills JC, Löbel U, et al. Cerebral neoplasms in L-2 hydroxyglutaric aciduria: 3 new cases and meta-analysis of literature data. Am J Neuroradiol. 2012;33:940-943.

37. Patay Z, Orr BA, Shulkin BL, et al. Successive distinct high-grade gliomas in L-2-hydroxyglutaric aciduria. J Inherit Metab Dis. 2015;38:273-277.

815 38. Kranendijk M, Struys EA, Salomons GS, et al. Progress in understanding 2-hydroxyglutaric acidurias. J Inherit Metab Dis. 2012;35:571-587.

39. Bardella C, Al-Dalahmah O, Krell D, et al. Expression of Idh1R132H in the murine subventricular zone stem cell niche recapitulates features of early gliomagenesis. Cancer Cell. 2016;30:578-594.

820 40. Sasaki M, Knobbe CB, Itsumi M, et al. D-2-hydroxyglutarate produced by mutant IDH1 perturbs collagen maturation and basement membrane function. Genes Dev. 2012;26:2038-2049.

41. Watanabe T, Nobusawa $S$, Kleihues $P$, et al. IDH1 mutations are early events in the development of astrocytomas and oligodendrogliomas. Am J Pathol. 2009;174:1149-1153.

42. Lai A, Kharbanda S, Pope WB, et al. Evidence for sequenced molecular evolution of IDH1 mutant glioblastoma from a distinct cell of origin. J Clin Oncol. 2011;29:4482-4490.

43. Johnson BE, Mazor T, Hong C, et al. Mutational analysis reveals the origin and therapy-driven evolution of recurrent glioma. Science. 2014;343:189-193.
44. Wakimoto H, Tanaka S, Curry WT, et al. Targetable signaling pathway mutations are associated with malignant phenotype in IDH-mutant gliomas. Clin Cancer Res. 2014;20:2898-2909.

45. Johannessen T-CA, Mukherjee J, Viswanath P, et al. Rapid conversion of mutant IDH1 from driver to passenger in a model of human gliomagenesis. Mol Cancer Res. 2016;14:976-983.

46. Mazor T, Chesnelong C, Pankov A, et al. Clonal expansion and epigenetic reprogramming following deletion or amplification of mutant IDH1. Proc Natl Acad Sci U. S. A. 2017;114:10743-10748.

47. Flavahan WA, Drier $Y$, Liau BB, et al. Insulator dysfunction and oncogene activation in IDH mutant gliomas. Nature. 2016;529:110-114.

- This paper shows that IDH mutation induces hypermethylation at CCCTC-binding factor (CTCF)-binding sites, resulting in the loss of insulation between topological domains and aberrant gene activation (in particular, a constitutive enhancer activates PDGFRA gene). It suggests a general mechanism of tumorigenesis induced by the disruption of chromosomal topology and subsequent aberrant regulatory interactions that induce oncogene expression.

48. Modrek AS, Golub D, Khan T, et al. Low-grade astrocytoma mutations in IDH1, P53, and ATRX cooperate to block differentiation of human neural stem cells via repression of SOX2. Cell Rep. 2017;21:1267-1280.

49. Phan K, Ng W, Lu VM, et al. Association between IDH1 and IDH2 mutations and preoperative seizures in patients with low-grade versus high-grade glioma: a systematic review and meta-analysis. World Neurosurg. 2018;111:e539-e545.

50. Kölker S, Pawlak V, Ahlemeyer B, et al. NMDA receptor activation and respiratory chain complex $V$ inhibition contribute to neurodegeneration in d-2-hydroxyglutaric aciduria. Eur J Neurosci. 2002;16:21-28.

51. Chen $\mathrm{H}$, Judkins J, Thomas $\mathrm{C}$, et al. Mutant IDH1 and seizures in patients with glioma. Neurology. 2017;88:1805-1813.

52. Amankulor NM, Kim Y, Arora S, et al. Mutant IDH1 regulates the tumor-associated immune system in gliomas. Genes Dev. 2017;31:774-786.

53. Berghoff AS, Kiesel B, Widhalm G, et al. Correlation of immune phenotype with IDH mutation in diffuse glioma. Neuro Oncol. 2017;19:1460-1468.

54. Kohanbash G, Carrera DA, Shrivastav S, et al. Isocitrate dehydrogenase mutations suppress STAT1 and CD8+ T cell accumulation in gliomas. J Clin Invest. 2017;127:1425-1437.

55. Zhang L, Sorensen MD, Kristensen BW, et al. D-2-hydroxyglutarate is an intercellular mediator in IDH-Mutant gliomas inhibiting complement and T cells. Clin Cancer Res. 2018. [cited 2018 Oct 20]. Available at http://clincancerres.aacrjournals.org/lookup/doi/10. 1158/1078-0432.CCR-17-3855

56. Coffelt SB, Wellenstein MD, de Visser KE. Neutrophils in cancer: neutral no more. Nat Rev Cancer. 2016;16:431-446.

57. Zhang $X$, Rao A, Sette $P$, et al. IDH mutant gliomas escape natural killer cell immune surveillance by downregulation of NKG2D ligand expression. Neuro Oncol. 2016;18:1402-1412.

58. Bunse $L$, Pusch $S$, Bunse $T$, et al. Suppression of antitumor $T$ cell immunity by the oncometabolite (R)-2-hydroxyglutarate. Nat Med. 2018;24:1192-1203.

-. This paper show thatD2HG is taken up by $T$ cells, resulting in suppression of $\mathrm{T}$ cell activity. Antitumor immunity induced by IDH1-specific vaccine or checkpoint inhibition can be improved by inhibition of the neomorphic enzymatic function of mutant IDH1, providing a strong rational for combining these two approaches in a clinical setting.

59. Zhang $X$, Zhu S, Li T, et al. Targeting immune checkpoints in malignant glioma. Oncotarget. 2016;8:7157-7174.

60. Röver LK, Gevensleben H, Dietrich J, et al. PD-1 (PDCD1) promoter methylation is a prognostic factor in patients with diffuse lower-grade gliomas harboring isocitrate dehydrogenase (IDH) mutations. EBioMedicine. 2018;28:97-104. 
61. Bleeker FE, Atai NA, Lamba S, et al. The prognostic IDH1(R132) mutation is associated with reduced NADP+-dependent IDH activity in glioblastoma. Acta Neuropathol. 2010;119:487-494.

62. Molenaar RJ, Botman D, Smits MA, et al. Radioprotection of IDH1-Mutated cancer cells by the IDH1-Mutant inhibitor AGI-5198. Cancer Res. 2015;75:4790-4802.

63. Labussière $M$, Idbaih $A$, Wang $X-W$, et al. All the $1 p 19 q$ codeleted gliomas are mutated on IDH1 or IDH2. Neurology. 2010;74:1886-1890.

64. Houillier C, Wang X, Kaloshi G, et al. IDH1 or IDH2 mutations predict longer survival and response to temozolomide in low-grade gliomas. Neurology. 2010;75:1560-1566.

65. Cancer Genome Atlas Research Network, Brat DJ, Verhaak RGW, et al.. Comprehensive, integrative genomic analysis of diffuse lower-grade gliomas. N Engl J Med. 2015;372:2481-2498.

915 66. Suzuki H, Aoki K, Chiba K, et al. Mutational landscape and clonal architecture in grade II and III gliomas. Nat Genet. 2015;47:458-468.

67. Chan AK-Y, Yao Y, Zhang Z, et al. Combination genetic signature stratifies lower-grade gliomas better than histological grade. Oncotarget. 2015;6:20885-20901.

920 68. Louis DN, Perry A, Reifenberger G, et al. The 2016 World Health Organization classification of tumors of the central nervous system: a summary. Acta Neuropathol. 2016;131:803-820.

69. Tabouret E, Nguyen AT, Dehais $C$, et al. Prognostic impact of the 2016 WHO classification of diffuse gliomas in the French POLA cohort. Acta Neuropathol. 2016;132:625-634.

70. Reuss DE, Mamatjan Y, Schrimpf D, et al. IDH mutant diffuse and anaplastic astrocytomas have similar age at presentation and little difference in survival: a grading problem for WHO. Acta Neuropathol. 2015;129:867-873.

930 71. Shirahata M, Ono T, Stichel D, et al. Novel, improved grading system(s) for IDH-mutant astrocytic gliomas. Acta Neuropathol. 2018;136:153-166.

72. Alentorn A, Dehais C, Ducray F, et al. Allelic loss of $9 p 21.3$ is a prognostic factor in $1 p / 19 q$ codeleted anaplastic gliomas. Neurology. 2015;85:1325-1331.

73. Figarella-Branger D, Mokhtari K, Dehais C, et al. Mitotic index, microvascular proliferation, and necrosis define 3 pathological subgroups of prognostic relevance among $1 \mathrm{p} / 19 \mathrm{q}$ co-deleted anaplastic oligodendrogliomas. Neuro Oncol. 2016;18:888-890.

94074 . Buckner JC, Shaw EG, Pugh SL, et al. Radiation plus procarbazine, CCNU, and vincristine in low-grade glioma. N Engl J Med. 2016;374:1344-1355.

75. Cairncross JG, Wang M, Jenkins RB, et al. Benefit from procarbazine, lomustine, and vincristine in oligodendroglial tumors is associated with mutation of IDH. J Clin Oncol. 2014;32:783-790.

76. van Den Bent MJ, Brandes AA, Taphoorn MJB, et al. Adjuvant procarbazine, lomustine, and vincristine chemotherapy in newly diagnosed anaplastic oligodendroglioma: long-term follow-up of EORTC brain tumor group study 26951. J Clin Oncol. 2013;31:344-350.

77. Hegi ME, Diserens A-C, Gorlia T, et al. MGMT gene silencing and benefit from temozolomide in glioblastoma. $\mathrm{N}$ Engl J Med. 2005;352:997-1003.

78. Chen F, Bian K, Tang $\mathrm{Q}$, et al. Oncometabolites $\mathrm{d}$ - and I-2-hydroxyglutarate inhibit the AlkB family DNA repair enzymes under physiological conditions. Chem Res Toxicol. 2017;30:1102-1110.

79. Wang $\mathrm{P}, \mathrm{Wu} J, \mathrm{Ma} S$, et al. Oncometabolite D-2-hydroxyglutarate inhibits ALKBH DNA repair enzymes and sensitizes IDH mutant cells to alkylating agents. Cell Rep. 2015;13:2353-2361.

80. Sulkowski PL, Corso CD, Robinson ND, et al. 2-hydroxyglutarate produced by neomorphic IDH mutations suppresses homologous recombination and induces PARP inhibitor sensitivity. Sci Transl Med. 2017;9.

965 - This paper presents one of the best examples of synthetic lethality in the context of IDH mutation. IDH mutation induces a homologous recombination (HR) defect that renders tumor cells exquisitely sensitive to poly(adenosine 5'-diphosphate- ribose) polymerase (PARP) inhibitors. This selective vulnerability is lost by treatment with IDH inhibitors.

81. Setty $P$, Hammes J, Rothämel $T$, et al. A pyrosequencing-based assay for the rapid detection of IDH1 mutations in clinical samples. J Mol Diagn. 2010;12:750-756.

82. Felsberg J, Wolter $M$, Seul $H$, et al. Rapid and sensitive assessment of the IDH1 and IDH2 mutation status in cerebral gliomas based on DNA pyrosequencing. Acta Neuropathol. 2010;119:501-507.

83. Horbinski C, Kelly L, Nikiforov YE, et al. Detection of IDH1 and IDH2 mutations by fluorescence melting curve analysis as a diagnostic tool for brain biopsies. J Mol Diagn. 2010;12:487-492.

84. Catteau A, Girardi H, Monville F, et al. A new sensitive PCR assay for one-step detection of $12 \mathrm{IDH} 1 / 2$ mutations in glioma. Acta Neuropathol Commun. 2014;2:58.

85. Boisselier $B$, Marie $Y$, Labussière $M$, et al. COLD PCR HRM: a highly sensitive detection method for IDH1 mutations. Hum Mutat. 2010;31:1360-1365.

86. Wang J, Zhao Y, Li J, et al. IDH1 mutation detection by droplet digital PCR in glioma. Oncotarget. 2015;6:39651-39660.

87. Perizzolo $M$, Winkfein $B$, Hui $S$, et al. IDH mutation detection in formalin-fixed paraffin-embedded gliomas using multiplex PCR and single-base extension. Brain Pathol. 2012;22:619-624.

88. Ramkissoon SH, Bi WL, Schumacher SE, et al. Clinical implementation of integrated whole-genome copy number and mutation profiling for glioblastoma. Neuro Oncol. 2015;17:1344-1355.

89. Sahm F, Schrimpf D, Jones DTW, et al. Next-generation sequencing in routine brain tumor diagnostics enables an integrated diagnosis and identifies actionable targets. Acta Neuropathol. 2016;131:903-910.

90. Nikiforova MN, Wald Al, Melan MA, et al. Targeted next-generation sequencing panel (GlioSeq) provides comprehensive genetic profiling of central nervous system tumors. Neuro Oncol. 2016;18:379-387.

91. Zacher A, Kaulich K, Stepanow S, et al. Molecular diagnostics of gliomas using next generation sequencing of a glioma-tailored gene panel. Brain Pathol. 2017;27:146-159.

92. Dubbink HJ, Atmodimedjo PN, Kros JM, et al. Molecular classification of anaplastic oligodendroglioma using next-generation sequencing: a report of the prospective randomized EORTC Brain Tumor Group 26951 Phase III Trial. Neuro Oncol. 2016;18:388-400.

93. Capper D, Weissert S, Balss J, et al. Characterization of R132H mutation-specific IDH1 antibody binding in brain tumors. Brain Pathol. 2010;20:245-254.

94. Kato $Y$, Jin $G$, Kuan $C-T$, et al. A monoclonal antibody IMab-1 specifically recognizes IDH1R132H, the most common glioma-derived mutation. Biochem Biophys Res Commun. 2009;390:547-551.

95. Zou Y, Bai HX, Wang Z, et al. Comparison of immunohistochemistry and DNA sequencing for the detection of $\mathrm{IDH} 1$ mutations in gliomas. Neuro Oncol. 2015;17:477-478.

96. Beiko J, Suki D, Hess KR, et al. IDH1 mutant malignant astrocytomas are more amenable to surgical resection and have a survival benefit associated with maximal surgical resection. Neuro Oncol. 2014; 16:81-91.

97. Shankar GM, Francis JM, Rinne ML, et al. Rapid intraoperative molecular characterization of glioma. JAMA Oncol. 2015;1:662-667.

98. Kanamori $M$, Kikuchi $A$, Watanabe $M$, et al. Rapid and sensitive intraoperative detection of mutations in the isocitrate dehydrogenase 1 and 2 genes during surgery for glioma. J Neurosurg. 2014;120:1288-1297.

99. Pirro V, Alfaro CM, Jarmusch AK, et al. Intraoperative assessment of tumor margins during glioma resection by desorption electrospray ionization-mass spectrometry. Proc Natl Acad Sci. 2017;201706459.

100. Santagata S, Eberlin LS, Norton I, et al. Intraoperative mass spectrometry mapping of an onco-metabolite to guide brain tumor surgery. Proc Natl Acad Sci. 2014;111:11121-11126.

101. Longuespée $R$, Wefers AK, De Vita $E$, et al. Rapid detection of 2-hydroxyglutarate in frozen sections of IDH mutant tumors by MALDI-TOF mass spectrometry. Acta Neuropathol Commun. 
2018;6 [cited 2018 Oct 22]. [Internet] available at https://actaneur ocomms.biomedcentral.com/articles/10.1186/s40478-018-0523-3

102. Uckermann O, Juratli TA, Galli R, et al. Optical analysis of glioma: Fourier-transform infrared spectroscopy reveals the IDH1 mutation status. Clin Cancer Res. 2018;24:2530-2538.

103. Uckermann $\mathrm{O}$, Yao $\mathrm{W}$, Juratli TA, et al. 2018. IDH1 mutation in human glioma induces chemical alterations that are amenable to optical Raman spectroscopy. J Neurooncol. Epub ahead of print. [cited 2018 Oct 22]. DOI:10.1007/s11060-018-2883-8

104. Rohle D, Popovici-Muller J, Palaskas N, et al. An inhibitor of mutant IDH1 delays growth and promotes differentiation of glioma cells. Science. 2013;340:626-630.

1050 105. Andronesi OC, Kim GS, Gerstner E, et al. Detection of 2-hydroxyglutarate in IDH-mutated glioma patients by in vivo spectral-editing and $2 \mathrm{D}$ correlation magnetic resonance spectroscopy. Sci Transl Med. 2012;4:116ra4.

106. Choi C, Ganji SK, DeBerardinis RJ, et al. 2-hydroxyglutarate detection by magnetic resonance spectroscopy in IDH-mutated patients with gliomas. Nat Med. 2012;18:624-629.

107. Pope WB, Prins RM, Albert Thomas $M$, et al. Non-invasive detection of 2-hydroxyglutarate and other metabolites in IDH1 mutant glioma patients using magnetic resonance spectroscopy. J Neurooncol. 2012;107:197-205.

108. Emir UE, Larkin SJ, de Pennington N, et al. Non-invasive quantification of 2-hydroxyglutarate in human gliomas with IDH1 and IDH2 mutations. Cancer Res. 2016;76:43-49.

109. Leather T, Jenkinson MD, Das K, et al. Magnetic resonance spectroscopy for detection of 2-hydroxyglutarate as a biomarker for IDH mutation in gliomas. Metabolites. 2017;7:E29.

110. Branzoli F, Di Stefano AL, Capelle L, et al. Highly specific determination of IDH status using edited in vivo magnetic resonance spectroscopy. Neuro Oncol. 2018;20:907-916.

1070 - This paper presents a highly sensitive and specific non-invasive method for D2HG detection by MRI spectroscopy, providing a useful tool for non-invasive determination of IDH status.

111. de la Fuente $\mathrm{Ml}$, Young RJ, Rubel J, et al. Integration of 2-hydroxyglutarate-proton magnetic resonance spectroscopy into clinical practice for disease monitoring in isocitrate dehydrogenase-mutant glioma. Neuro Oncol. 2016;18:283-290.

112. Tietze A, Choi C, Mickey B, et al. Noninvasive assessment of isocitrate dehydrogenase mutation status in cerebral gliomas by magnetic resonance spectroscopy in a clinical setting. J Neurosurg. 2018;128:391-398.

113. Intlekofer AM, Dematteo RG, Venneti $S$, et al. Hypoxia induces production of L-2-Hydroxyglutarate. Cell Metab. 2015;22:304-311.

114. Andronesi OC, Loebel F, Bogner W, et al. Treatment response assessment in IDH-Mutant glioma patients by noninvasive 3D functional spectroscopic mapping of 2-Hydroxyglutarate. Clin Cancer Res. 2016;22:1632-1641.

115. Choi C, Raisanen JM, Ganji SK, et al. Prospective longitudinal analysis of 2-Hydroxyglutarate magnetic resonance spectroscopy identifies broad clinical utility for the management of patients with IDH-Mutant glioma. J Clin Oncol. 2016;34:4030-4039.

116. Park YW, Han K, Ahn SS, et al. Prediction of IDH1-Mutation and $1 \mathrm{p} /$ 19q-Codeletion status using preoperative MR imaging phenotypes in lower grade gliomas. Am J Neuroradiol. 2018;39:37-42.

117. Villanueva-Meyer JE, Wood MD, Choi BS, et al. MRI features and IDH mutational status of grade II diffuse gliomas: impact on diagnosis and prognosis. AJR Am J Roentgenol. 2018;210:621-628.

118. Leu K, Ott GA, Lai A, et al. Perfusion and diffusion MRI signatures in histologic and genetic subtypes of WHO grade II-III diffuse gliomas. J Neurooncol. 2017;134:177-188.

1100 119. Kickingereder P, Sahm F, Radbruch A, et al. IDH mutation status is associated with a distinct hypoxia/angiogenesis transcriptome signature which is non-invasively predictable with rCBV imaging in human glioma. Sci Rep. 2015;5:16238.

120. Patel SH, Poisson LM, Brat DJ, et al. T2-FLAIR mismatch, an imaging biomarker for IDH and $1 p / 19 q$ status in lower-grade gliomas: a TCGA/TCIA project. Clin Cancer Res. 2017;23:6078-6085.
- This paper reports a new radiological sign, the T2-FLAIR mismatch sign, specific for IDH-mutant, $1 \mathrm{p} / 19 q$ non-codeleted tumors.

121. Broen MPG, Smits M, Wijnenga MMJ, et al. The T2-FLAIR mismatch sign as an imaging marker for non-enhancing IDH-mutant, $1 \mathrm{p} /$ 19q-intact lower grade glioma: a validation study. Neuro Oncol. 2018;20:1393-1399.

122. Lasocki A, Gaillard F, Gorelik A, et al. MRI features can predict $1 p / 19 q$ status in intracranial gliomas. Am J Neuroradiol. 2018;39:687-692.

123. Janin M, Mylonas E, Saada V, et al. Serum 2-hydroxyglutarate production in IDH1- and IDH2-mutated de novo acute myeloid leukemia: a study by the Acute Leukemia French Association Group. J Clin Oncol. 2014;32:297-305.

124. Borger DR, Goyal L, Yau T, et al. Circulating oncometabolite 2-hydroxyglutarate is a potential surrogate biomarker in patients with isocitrate dehydrogenase-mutant intrahepatic cholangiocarcinoma. Clin Cancer Res. 2014;20:1884-1890.

125. Capper D, Simon M, Langhans C-D, et al. 2-hydroxyglutarate concentration in serum from patients with gliomas does not correlate with IDH1/2 mutation status or tumor size. Int J Cancer. 2012;131:766-768.

126. Fathi AT, Nahed BV, Wander SA, et al. Elevation of urinary 2-hydroxyglutarate in IDH-Mutant glioma. Oncologist. 2016;21:214-219.

127. Lombardi G, Corona G, Bellu L, et al. Diagnostic value of plasma and urinary 2-hydroxyglutarate to identify patients with isocitrate dehydrogenase-mutated glioma. Oncologist. 2015;20:562-567.

128. Kalinina J, Ahn J, Devi NS, et al. Selective detection of the D-enantiomer of 2-hydroxyglutarate in the CSF of glioma patients with mutated isocitrate dehydrogenase. Clin Cancer Res. 2016;22:6256-6265.

129. Best MG, Sol N, Zijl S, et al. Liquid biopsies in patients with diffuse glioma. Acta Neuropathol. 2015;129:849-865.

130. Fontanilles $M$, Duran-Peña $A$, Idbaih A. Liquid biopsy in primary brain tumors: looking for stardust! Curr Neurol Neurosci Rep. 2018;18:13.

131. Wang J, Zhao Y, Li J, et al. IDH1 mutation detection by droplet digital PCR in glioma. Oncotarget. 2015;6:39651-39660.

132. Boisselier B, Gállego Pérez-Larraya J, Rossetto M, et al. Detection of IDH1 mutation in the plasma of patients with glioma. Neurology. 2012;79:1693-1698.

133. Martínez-Ricarte F, Mayor R, Martínez-Sáez E, et al. Molecular diagnosis of diffuse gliomas through sequencing of cell-free circulating tumor DNA from cerebrospinal fluid. Clin Cancer Res. 2018;24:2812-2819.

134. Chen WW, Balaj L, Liau LM, et al. BEAMing and droplet digital PCR analysis of mutant IDH1 mRNA in glioma patient serum and cerebrospinal fluid extracellular vesicles. Mol Ther Nucleic Acids. 2013;2:e109.

135. Pusch S, Krausert S, Fischer V, et al. Pan-mutant IDH1 inhibitor BAY 1436032 for effective treatment of IDH1 mutant astrocytoma in vivo. Acta Neuropathol. 2017;133:629-644.

136. Kopinja J, Sevilla RS, Levitan D, et al. A brain penetrant mutant IDH1 inhibitor provides in vivo survival benefit. Sci Rep. 2017;7:13853.

137. Popovici-Muller J, Lemieux RM, Artin E, et al. Discovery of AG-120 (Ivosidenib): a first-in-class mutant IDH1 inhibitor for the treatment of IDH1 mutant cancers. ACS Med Chem Lett. 2018;9:300-305.

138. Cho YS, Levell JR, Liu G, et al. Discovery and evaluation of clinical candidate IDH305, a brain penetrant mutant IDH1 inhibitor. ACS Med Chem Lett. 2017;8:1116-1121.

139. Machida Y, Ogawara Y, Ichimura K, et al. Abstract 3101: the mutant IDH1 inhibitor prevents growth of glioblastoma with IDH1 mutation in patient-derived xenograft (PDX) model. Cancer Res. 2016;76:3101.

140. Tateishi $\mathrm{K}$, Wakimoto $\mathrm{H}$, lafrate $\mathrm{AJ}$, et al. Extreme vulnerability of IDH1 mutant cancers to NAD+ depletion. Cancer Cell. 2015;28:773-784.

141. Turcan S, Makarov V, Taranda J, et al. Mutant-IDH1-dependent chromatin state reprogramming, reversibility, and persistence. Nat Genet. 2018;50:62-72. 
- This paper showed that several epigenetic changes induced by D2HG are irreversible, while other are not. This has important implications for optimizing therapeutic strategies.

142. DiNardo CD, Stein EM, de Botton S, et al. Durable remissions with ivosidenib in IDH1-Mutated relapsed or refractory AML. N Engl J Med. 2018;378:2386-2398.

1185 143. Mellinghoff IK, Touat M, Maher E, et al. ACTR-46. AG120, a first-inclass mutant idh1 inhibitor in patients with recurrent or progressive idh1 mutant glioma: results from the phase 1 glioma expansion cohorts. Neuro Oncol. 2016;18:vi12.

144. Mellinghoff $I$, Penas-Prado $M$, Peters $M$, et al. Phase 1 study of AG-881, an inhibitor of mutant IDH1/IDH2, in patients with advanced IDH-mutant solid tumors, including glioma. J Clin Oncol 2018;36:abstr 2002.

145. Borodovsky A, Salmasi V, Turcan S, et al. 5-azacytidine reduces methylation, promotes differentiation and induces tumor regression in a patient-derived IDH1 mutant glioma xenograft. Oncotarget. 2013;4:1737-1747.

146. Turcan S, Fabius AWM, Borodovsky A, et al. Efficient induction of differentiation and growth inhibition in IDH1 mutant glioma cells by the DNMT inhibitor decitabine. Oncotarget. 2013;4:1729-1736.

147. Schumacher $T$, Bunse $L$, Pusch $S$, et al. A vaccine targeting mutant IDH1 induces antitumour immunity. Nature. 2014;512:324-327.

148. Pellegatta S, Valletta L, Corbetta $C$, et al. Effective immuno-targeting of the IDH1 mutation $\mathrm{R} 132 \mathrm{H}$ in a murine model of intracranial glioma. Acta Neuropathol Commun. 2015;3:4.

149. Platten $M$, Schilling $D$, Bunse $T$, et al. A mutation-specific peptide vaccine targeting IDH1R132H in patients with newly diagnosed malignant astrocytomas: a first-in-man multicenter phase I clinical trial of the German Neurooncology Working Group (NOA-16). J Clin Oncol. 2016;34:TPS2082. vaccine targeting IDH1R132H in patients with newly diagnosed

malignant astrocytomas: a first-in-man multicenter phase I clinical trial of the German Neurooncology Working Group (NOA-16). J Clin Oncol 2018;36:abstr 2001.

151. Archer GE, Reap E, Norberg P, et al. IDH1 mutations as a immunotherapeutic target for brain tumors. Neuro Oncol. 2014;16:iii40.

152. Lu Y, Kwintkiewicz J, Liu Y, et al. Chemosensitivity of IDH1-Mutated gliomas due to an impairment in PARP1-mediated DNA repair. Cancer Res. 2017;77:1709-1718.

153. Feng J, Yan P-F, Zhao H, et al. Inhibitor of nicotinamide phosphoribosyl transferase sensitizes glioblastoma cells to temozolomide via activating ROS/JNK signaling pathway. BioMed Res Int. 2016;2016:1450843.

154. Karpel-Massler G, Ishida CT, Bianchetti $E$, et al. Induction of synthetic lethality in IDH1-mutated gliomas through inhibition of Bcl-xL. Nat Commun. 2017;8:1067.

155. Seltzer MJ, Bennett BD, Joshi AD, et al. Inhibition of glutaminase preferentially slows growth of glioma cells with mutant IDH1. Cancer Res. 2010;70:8981-8987.

156. van Lith SAM, Navis AC, Verrijp K, et al. Glutamate as chemotactic fuel for diffuse glioma cells: are they glutamate suckers? Biochim Biophys Acta. 2014;1846:66-74.

157. Khurshed $M$, Molenaar RJ, Lenting $K$, et al. In silico gene expression analysis reveals glycolysis and acetate anaplerosis in IDH1 wild-type glioma and lactate and glutamate anaplerosis in IDH1-mutated glioma. Oncotarget. 2017;8:49165-49177.

158. Melin BS, Barnholtz-Sloan JS, Wrensch MR, et al. Genome-wide association study of glioma subtypes identifies specific differences in genetic susceptibility to glioblastoma and non-glioblastoma tumors. Nat Genet. 2017;49:789-794.

159. Labreche $K$, Kinnersley B, Berzero $G$, et al. Diffuse gliomas classified by $1 p / 19 q$ co-deletion, TERT promoter and IDH mutation status are associated with specific genetic risk loci. Acta Neuropathol. 2018;135:743-755. 